\title{
Central and Peripheral Nervous System Disorders Following Ivermectin Mass Administration: A Descriptive Study Based on the Democratic Republic of Congo Pharmacovigilance System
}

\author{
Didier Nzolo ${ }^{1,2,3}$ - Francis Anto ${ }^{1}$ Sarah Hailemariam ${ }^{2,3} \cdot$ Didier Bakajika $^{4}$. \\ Daniel Muteba $^{5}$ - Jean-Claude Makenga ${ }^{6}$ - Gautier Mesia ${ }^{2,3}$ - Celestin Nsibu ${ }^{2,3,7}$. \\ Samuel Mampunza ${ }^{2,3,8} \cdot$ Gaston Tona $^{2,3}$
}

Published online: 9 June 2017

(c) The Author(s) 2017. This article is an open access publication

\begin{abstract}
Introduction The mainstay of onchocerciasis control currently is mass administration of ivermectin; however, this may be associated with serious adverse events, including deaths, when administered in areas where onchocerciasis and loiasis are co-endemic.

Objectives The objective of the current study was to describe the central and peripheral nervous system disorders that occurred after mass administration of ivermectin in Democratic Republic of Congo (DRC).

Methods This is a retrospective descriptive study involving a review of data on adverse events related to mass administration of ivermectin. Data on reported serious adverse events following mass administration of iver-
\end{abstract}

Francis Anto

fanto@ug.edu.gh

Didier Nzolo

didiernzolo@yahoo.fr

Sarah Hailemariam

sarahnehema@gmail.com

Didier Bakajika

dbakajika@sightsavers.org

Daniel Muteba

danielmuteba2002@yahoo.fr

Jean-Claude Makenga

jcmakebof@yahoo.fr

Gautier Mesia

Mesia.kahunu@unikin.ac.cd

Celestin Nsibu

celnsibu@hotmail.fr

Samuel Mampunza

smmampunza@hotmail.com

Gaston Tona

tonalutete@gmail.com mectin in the DRC were extracted from the World Health Organization (WHO) Global individual case safety report (ICSR) database (VigiBase). The review covered the period 2009-2013 and focused on central and peripheral nervous system disorders. Relevant demographic, clinical, and parasitological data, including age, sex, area of residence, adverse events, and parasite density were extracted. Descriptive statistics were analyzed using Stata 12.

Results A total of 52 ICSRs related to ivermectin intake were available in VigiBase, with $51(98.1 \%)$ from the Province of Equateur. All patients had central and peripheral nervous system disorders; $25(48.1 \%)$ had altered mental status. Of these, $23(92.0 \%)$ satisfied the criteria for "probable/possible Loa loa encephalopathy

1 School of Public Health, University of Ghana, Legon, Accra, Ghana

2 Unit of Clinical Pharmacology and Pharmacovigilance, University of Kinshasa, Kinshasa, Democratic Republic of Congo

3 Centre National de Pharmacovigilance, Kinshasa, Democratic Republic of Congo

4 Sightsavers, Hayward Heath, UK

5 Programme Nationale de Lutte contre l'Onchocercose, Kinshasa, Democratic Republic of Congo

6 Ecole de Santé Publique, Université Libre de Bruxelles, Brussels, Belgium

7 Cliniques Universitaires de Kinshasa, Kinshasa, Democratic Republic of Congo

8 Centre Neuro-Psycho-Pathologique, Kinshasa, Democratic Republic of Congo 
temporally related to mectizan ${ }^{\circledR}$ " (PLERM). The most frequent nervous system disorders among patients with PLERM were coma (74\%), stupor (30\%), headache (22\%), and abnormal gait (22\%). There were, on average, 2149.1 microfilariae per $\mathrm{ml}(\mathrm{mf} / \mathrm{ml})$ in peripheral blood [95\% confidence interval (CI) 463.6-3834.6; $n=23$ ]. Posttreatment, $61 \%$ of PLERM cases had $<1000$ L. loa $\mathrm{mf} / \mathrm{ml}$ of blood. One patient had microfilariae in the cerebrospinal fluid rather than the peripheral blood. We found $21.4 \%$ coinfection with Plasmodium falciparum and $4 \%$ mortality. Conclusion PLERM may occur at even low peripheral blood concentrations of microfilaria.

\section{Key Points}

The effects of central nervous system disorders following ivermectin administration occurred in several systems in the body.

Loa loa encephalopathy related to mectizan may result at low blood levels of microfilaria.

\section{Introduction}

Onchocerciasis is a neglected disease and one of the two most important filarial infections that may lead to severe pathology in humans $[1,2]$. It is caused by the parasite Onchocerca volvulus and transmitted by infected blackflies of the genus Simulium. The majority of cases of onchocerciasis occur in sub-Saharan Africa within the equatorial region. An estimated 102 million people are at risk of onchocerciasis in 20 African countries [1,3]. The Democratic Republic of Congo (DRC) is the largest filariasis endemic country in Africa, with 31.3 million people at risk of onchocerciasis $[4,5]$.

The two main problems associated with onchocerciasis are its ocular and cutaneous manifestations. Onchocercal ocular disease and blindness result from chronic infections, making onchocerciasis the world's second-leading cause of infectious blindness. On the skin, it causes severe itching, disfiguring skin lesions, and depigmentation $[3,6]$.

The current mainstay of onchocerciasis control is mass administration of ivermectin in endemic communities. Ivermectin is a macrocyclic lactone endectocide discovered in the mid-1970s and first used for the treatment of nematode and arthropod parasites in animals [6]. Since the 1980s, its use in humans for the treatment of filariasis, especially onchocerciasis, gave hope for the control and possible eradication of filariasis in humans [7]. However, one of its major problems is its association with serious adverse events (SAEs), including deaths when administered in regions where onchocerciasis and loiasis are co-endemic [8]. The issue of SAEs due to ivermectin is a threat to adherence to mass drug administration (MDA) and the control and elimination of disease in communities co-endemic with $O$. volvulus and Loa loa. MDAs were suspended from 2004 to 2006 in some health zones in the Equateur, Bas Congo, and Oriental provinces in the DRC because of ivermectin-associated SAEs in the Bas Congo and Oriental provinces in 2003 [5].

Unfortunately, very few reports are available from countries co-endemic with $O$. volvulus and L. loa [9] to describe the extent of the disorders and thus enable the design of strategies for safe administration of ivermectin for onchocerciasis or lymphatic filariasis in such regions [10]. For example between 1981 and 2001, only 207 SAEs were reported from seven African countries. Among them were 103 cases of encephalopathy, with 65 satisfying the definition of "probable or possible $L$. loa encephalopathy temporally related to treatment with mectizan ${ }^{\circledR}$ " (PLERM) $[11,12]$. Most of these SAEs $(85 \% ; 176 / 207)$ were reports from Cameroon, with only five (2.4\%) reported from the DRC $[8,11]$. Therefore, the purpose of the current report is to describe central and peripheral nervous system disorders following mass administration of ivermectin as captured by the DRC pharmacovigilance system in order to contribute to the clinical and epidemiological picture of L. loa encephalopathy in the country.

\section{Methods}

\subsection{Study Area}

The DRC is in Central Africa and covers an area of 2.3 million $\mathrm{km}^{2}$ and has a population of over 70 million. The DRC health system consists of the national level, 11 provinces, 52 health districts (HDs), and 515 health zones (HZs), which constitutes the operational level. At the HZ level, healthcare is provided at either university hospitals, provincial general referral hospitals, referral hospitals, referral health centers, health centers, or as communitybased care. Onchocerciasis is prevalent in all 11 of the provinces of the DRC at different levels of endemicity and among populations living in remote areas along rivers. Estimates in 2012 revealed that 41,618 communities with a total population of 31.3 million are at risk of infection, with 14 million already infected [5].

MDA is undertaken in meso- and hyperendemic communities. A single dose of ivermectin (Mectizan ${ }^{\circledR}$ ) $150 \mathrm{mg} /$ $\mathrm{kg}$ is given annually, monitored by the National Onchocerciasis program (PNLO) and essentially funded by the African Programme for Onchocerciasis Control 
(APOC). Two strategies are used to increase the coverage of the MDA: community-directed treatment with ivermectin (CDTI) and health-worker based treatment. By 2012, about 119,340 community-directed distributors and 13,493 health worker-based treatment centers were established in a total of 39,100 communities, leading to geographical and therapeutic coverage of 93.9 and $74.2 \%$, respectively.

Between 2001 and 2012, CDTI activities covered the following provinces: Occidental Kasai, Oriental Kasai (Sankuru), Oriental Province (Uele, Tshopo, North Ituri, and South Ituri), Bandundu (Bandundu), Katanga (North Katanga, South Katanga, and Lualaba), Equateur (Tshuapa, North Ubangi, South Ubangi, Mongala, and EquateurKiri), North Kivu (Rutshuru-Goma, Butembo-Beni, and Masisi-Walikale), Maniema (Kasongo and Lubutu), Kinshasa, and Bas-Congo (Bas-Congo/Kinshasa project). In 2004, CDTI activities in Bas-Congo, Kinshasa, Tshopo, and Uele were suspended because of SAEs in Bas-Congo [5]. From 2008 to 2012, the DRC had 21 operational CDTI activity areas, with 16 of these areas co-endemic for onchocerciasis and loiasis.

\subsection{Democratic Republic of Congo (DRC) Pharmacovigilance and Data Management System}

The PNLO employs a passive surveillance system for the reporting of SAEs following ivermectin MDA and uses SAE reporting forms provided by The Mectizan ${ }^{\circledR}$ Donation Program as individual case safety reports (ICSRs). ICSRs contain information on patient demographics, drugs administered, SAEs, and laboratory results, including levels of $L$. loa microfilariae $(\mathrm{mf})$ in blood and/or cerebrospinal fluid (CSF) on a post-treatment basis. Searches for microfilariae in blood are performed in the field using a calibrated thick blood smear test. The data collection covers all health system levels from the operational to the national level. At the national level, the PNLO collaborates with the DRC National Pharmacovigilance Center for data management on SAEs related to ivermectin. The CNPV makes available the ICSRs submitted by health workers during ivermectin MDA. The CNPV receives completed ICSRs, performs analysis and causality assessment, and enters data into the World Health Organization (WHO) Global ICSR database (VigiBase).

Before data entry, junior assistants perform two analyses of the ICSRs: the first aims to assess completeness and the second is for causality assessment. The software used to enter data into VigiBase is called VigiFlow. Senior assistants check the ICSRs entered into VigiFlow for discrepancies then commit and send the ICRS from VigiFlow into VigiBase. In VigiFlow, reactions are registered using the
WHO adverse drug reaction terminology. Preferred terms (PTs) are generally used to describe the reported adverse event. The software automatically generates the corresponding system and organ class (SOC) terms. Feedback is provided to the PNLO and, if necessary, to the health worker who reported any specific cases.

\subsection{Study Design}

This was a retrospective descriptive study involving the review of data on adverse events related to mass administration of ivermectin extracted from the DRC pharmacovigilance system, which has been registered into the WHO Global ICSR database (VigiBase). The review covered the period 2009-2013 and focused on reported central and peripheral nervous system disorders.

\subsection{Data Extraction}

Data were extracted into a Microsoft ${ }^{\circledR}$ Excel spreadsheet and PDF file from VigiBase using VigiLyze software in December 2013. Search criteria in VigiBase included the substance ivermectin as the drug and DRC as the country. For supplementary information missing from VigiBase, we used an Excel spreadsheet generated via the 'search and statistics' function of VigiFlow. The following search criteria were used in VigiFlow: drug (ivermectin); drug operator (or); drug level (active substance); reaction level (preferred); include concomitant (yes). When further precision was needed, we consulted either the ICSRs, which were the main primary source, or the internal printout generated from the 'search and statistics' report list. For analysis of adverse events, only SOC terms and PTs were considered. In SOC, central and peripheral nervous system disorders were separated from other system and organ disorders.

\subsection{Definitions}

The International Conference on Harmonization (ICH) Guideline for Good Clinical Practice defines an SAE as any untoward medical occurrence that, at any dose, results in death, is life-threatening, requires hospitalization or prolongation of existing hospitalization, results in persistent or significant disability/incapacity, or is a congenital anomaly/birth defect. Central and peripheral nervous system disorders were automatically generated from the VigiFlow software. $L$. loa encephalopathy and PLERM were defined according to the latest adaptation, where 'probable' refers to a probable case of $L$. loa encephalopathy that contains the following elements: (1) encephalopathy (without seizures, usually with fever) in a person previously healthy and with no other underlying cause for encephalopathy, 
and (2) onset of progressive central nervous system symptoms and signs within 7 days of treatment with ivermectin; illness progressing to coma without remission, and (3) peripheral blood L. loa $>10,000 \mathrm{mf} / \mathrm{ml}$ pre-treatment or $>1000 \mathrm{mf} / \mathrm{ml}$ within 1 month post-treatment or $>2700$ $\mathrm{mf} / \mathrm{ml}$ within 6 months of treatment; and/or L. loa microfilariae present in CSF within 1 month post-treatment [11].

\subsection{Data Analysis}

The generated Excel spreadsheet was exported into Stata version 12. Both Excel and Stata software were used for data analysis. Categorical variables were summarized as frequencies and percentages. Continuous variables were summarized as means with their $95 \%$ confidence interval (CI). For data that were not normally distributed, the median and interquartile ranges (IQRs) were provided when necessary. Statistics generated by the VigiLyze software were also used. If discrepancies were noticed when comparing results from VigiFlow and VigiBase, data from the VigiFlow 'search and statistics' were considered. Analysis was limited to descriptive statistics because of the small number of reports.

\subsection{Ethical Issues}

This retrospective data analysis was based on national pharmacovigilance system that had already collected and archived the data. Therefore, the consent of individual participants could not be sought. The current analysis did not include individual identifiers.

\section{Results}

\subsection{Distribution of Central and Peripheral Nervous System Disorders Following Ivermectin Administration}

As at December 2013, a total of 52 ICSRs had been entered into VigiBase by the CNPV of the DRC. A total of 17 cases were reported for 2009, 33 for 2010, and two for 2011. Most of the reports [48/52 (92.3\%)] were made by physicians, with other health professionals, mainly nurses, reporting four $(7.3 \%)$ of the cases. No records were available in the database for the years 2012 and 2013. Of the patients, $31(59.6 \%)$ were males, and the mean age was 35.7 years (95\% CI 32.4-39.1; range 20-73) (Table 1).

Almost all the reported cases [51/52 (98.1\%)] came from the province of Equateur; one (1.9\%) came from the Oriental province (Fig. 1). The cases from the province of Equateur consisted of 41 from North Ubangi (HZ of Loko 16, Businga 12, Boto 9, Gbadolite 3, and Yakoma 1) and ten from South Ubangi (HZ of Bominenge 2, Bwamanda and Ndage 4 cases each). The Oriental province case came from the HD of Dingila, HZ of Viadana.

\subsection{Reported Systems and Organs Disorders and Related Adverse Events}

Several system and organ disorders were reported among the 52 patients. All patients had central and peripheral nervous system disorders: 25 of the $52(48.1 \%)$ had altered mental status, with $23(92.0 \%)$ meeting the criteria for PLERM, and two had encephalopathy of unknown etiology. One of these two had no microfilariae in the blood, and data on L. loa microfilaremia were missing for the other. Of those with PLERM, 14 (60.9\%) were males, and the mean age was 37.3 years (95\% CI 31.1-43.6; range 20-73). The mean time of onset of symptoms was 2.6 days (95\% CI 2.1-3.2) for all cases $(n=52)$ and 2.4 days $(95 \%$ CI 2.0-2.9) for PLERM cases $(n=23)$ (Table 1).

The most reported system and organ disorders were general disorders [36/52 (69\%)], vascular extra-cardiac disorders [17/52 (33\%)], and urinary system disorders [12/ $52(23 \%)]$. There were six cases of psychiatric disorder (12\%) (Fig. 2).

The most reported central and peripheral nervous system disorders were headaches [22/52 (42.3\%)], coma [18/52 (34.6\%)], abnormal gait [17/52 (32.7\%)], and stupor [8/52 (15.4\%)]. Among the PLERM cases, the frequencies of these adverse events differed, with the most frequent being coma [17/23 (73.9\%)], followed by stupor [7/23 (30.4\%)], headache [5/23 (21.7\%)], and abnormal gait [5/23 (21.7\%)] (Table 2). Adverse events reported in other systems and organs were asthenia [24/52 (46.2\%)], conjunctival hemorrhage [17/52 (32.7\%)], fever [16/52 (30.8\%)], back pain [9/52 (17.3\%)], and urinary incontinence [9/52 (17.3\%)]. Psychiatric disorders reported included agitation, abnormal behavior, and personality disorders. Among patients with PLERM, the most reported conditions in other systems and organs were asthenia [10/23 (43.5\%)], conjunctival hemorrhage $[7 / 23(30.4 \%)]$, urinary incontinence $[7 / 23$ $(30.4 \%)]$, fever $[6 / 23(26.1 \%)]$, and myalgia $[3 / 23$ (13.0\%)] (Table 3).

\subsection{Parasitological Measurements and Patient Outcomes}

The mean concentration of $L$. loa microfilariae in the peripheral blood of all patients with central and peripheral nervous system disorders $(n=51)$ was $1743.6[95 \% \mathrm{CI}$ 938.5-2548.7; median 820 (IQR 220-2240)] and of PLERM cases $(n=23)$ was $2149.1 \quad[95 \% \quad$ CI 463.6-3834.6; median 600 (IQR 200-2540)]. Most of the patients $(55.8 \%$ of all cases and $60.9 \%$ of PLERM cases) 
Table 1 General information about patients, adverse events, and laboratory results

\begin{tabular}{|c|c|c|}
\hline \multirow[t]{2}{*}{ Variables } & \multicolumn{2}{|l|}{ Frequency $(\%)$} \\
\hline & All $(n=52)$ & PLERM $(n=23)$ \\
\hline \multicolumn{3}{|l|}{ Sex } \\
\hline Male & $31(59.6)$ & $14(60.9)$ \\
\hline Female & $19(36.5)$ & $9(39.1)$ \\
\hline Unknown & $2(3.9)$ & $0(0.0)$ \\
\hline Age (years) & $35.7(32.4-39.1)$ & $37.3(31.1-43.6)$ \\
\hline $15-29$ & $18(34.62)$ & $8(34.8)$ \\
\hline $30-44$ & $24(46.15)$ & $9(39.1)$ \\
\hline $45-59$ & $8(15.38)$ & $4(17.4)$ \\
\hline$\geq 60$ & $2(3.85)$ & $2(8.7)$ \\
\hline $\begin{array}{l}\text { Time to onset of } \\
\text { symptoms (days) }\end{array}$ & $2.6(2.1-3.2)$ & $2.4(2.0-2.9)$ \\
\hline $24-48 \mathrm{~h}$ & $29(55.8)$ & $11(47.8)$ \\
\hline $49-96 \mathrm{~h}$ & $20(38.5)$ & $12(52.2)$ \\
\hline 5-14 days & $3(5.8)$ & $0(0.0)$ \\
\hline SAE & $51(98.1)$ & $23(100.0)$ \\
\hline \multicolumn{3}{|l|}{$\begin{array}{l}\text { Seriousness } \\
\text { criteria }\end{array}$} \\
\hline Death & $3(5.8)$ & $1(4.4)$ \\
\hline Life threatening & $2(3.9)$ & $1(4.4)$ \\
\hline Hospitalized & $48(92.3)$ & $23(95.7)$ \\
\hline Disabling & $3(5.8)$ & $2(8.7)$ \\
\hline $\begin{array}{l}\text { Loa loa } \\
\text { microfilaremia } \\
(\mathrm{mf} / \mathrm{ml})\end{array}$ & $1743.6(938.5-2548.7)$ & $2149.1(463.6-3834.6)$ \\
\hline$<1000$ & $29(55.8)$ & $14(60.9)$ \\
\hline $1000-10,000$ & $20(38.5)$ & $8(34.8)$ \\
\hline$>10,000$ & $1(1.9)$ & $1(4.4)$ \\
\hline Unknown & $2(3.9)$ & $0(0.0)$ \\
\hline \multicolumn{3}{|l|}{$\begin{array}{l}\text { Presence of Loa } \\
\text { loa microfilariae } \\
\text { in CSF }\end{array}$} \\
\hline Present & $1(1.9)$ & $1(4.4)$ \\
\hline Absent & $1(1.9)$ & $1(4.4)$ \\
\hline Unknown & $50(96.2)$ & $21(91.3)$ \\
\hline \multicolumn{3}{|l|}{ Malaria infection } \\
\hline Positive & $3(5.8)$ & $1(4.4)$ \\
\hline Negative & $11(21.2)$ & $2(8.7)$ \\
\hline Unknown & $38(73.1)$ & $20(87.0)$ \\
\hline
\end{tabular}

Data are presented as $n(\%)$ or mean $(95 \% \mathrm{CI})$

$C I$ confidence interval, $C S F$ cerebrospinal fluid, $\mathrm{mf} / \mathrm{ml}$ microfilariae per milliliter, PLERM probable/possible Loa loa encephalopathy temporally related to mectizan, $S A E$ serious adverse event

had $<1000 \mathrm{mf} / \mathrm{ml}$ of peripheral blood. The CSF of two patients was examined microscopically, and one was found to be positive for $L$. loa microfilariae. However, this patient did not have microfilariae in the peripheral blood and was in a coma. A Giemsa-stained thick blood smear was performed for 14 patients; three (21.4\%) were found to be positive for Plasmodium falciparum infection. P. falciparum was found in one of three PLERM cases tested (Table 1). Of all patients, three (5.8\%) died and three (5.8\%) had disabilities; in PLERM cases, one $(4.4 \%)$ died and two $(8.7 \%)$ had disability (Table 1$)$.

\section{Discussion}

Data on reported adverse events following ivermectin MDA in the DRC were extracted from the National Pharmacovigilance System registered into VigiBase. The review focused on central and peripheral nervous system disorders. A total of 52 SAEs were included in the analysis. Almost all the reported cases $(98.1 \%)$ came from the province of Equateur, with one coming from the Oriental province. In total, 25 patients had altered mental status, 23 of whom met the criteria for PLERM.

A wide range of adverse events, including headache, coma, abnormal gait, stupor, asthenia, conjunctival hemorrhage, fever, back pain, urinary incontinence, and psychiatric disorders were reported, mainly in males [11, 12] following the administration of ivermectin. Similar adverse events have been reported in earlier research $[8,13,14]$. According to Boussinesq et al. [8], post-ivermectin L. loarelated SAEs are probably not due to a direct toxic effect of the drug, but the main factor associated with the risk of post-ivermectin SAEs is the L. loa microfilariae load. Central and peripheral nervous disorders, specifically $L$. loa encephalopathy, should be caused by the effect of $L$. loa microfilariae at the cerebral level after ivermectin administration [8].

These reports on adverse events were not distributed across all provinces in which ivermectin MDA took place but mainly from the province of Equateur, similar to findings of Twum-Danso [11] in Cameroon, where 97\% of 65 PLERM cases came from the southern part of the country. This focality of SAEs has been attributed to coendemicity of $O$. volvulus and $L$. loa [15], with the main risk factor being a high intensity of Loa microfilariae [8] and taking the drug for the first time [16].

Although this predominance of reporting from Equator may be due to a poor national spontaneous adverse event reporting system with uneven reporting across provinces, it remains very important to know the localities in which people are more likely to experience SAEs following ivermectin MDA. This will enable health personnel to adequately prepare and direct attention to such areas to ensure comprehensive care when adverse events occur. According to Kelly-Hope et al. [15], there is significant geographical overlap between low onchocerciasis and high 


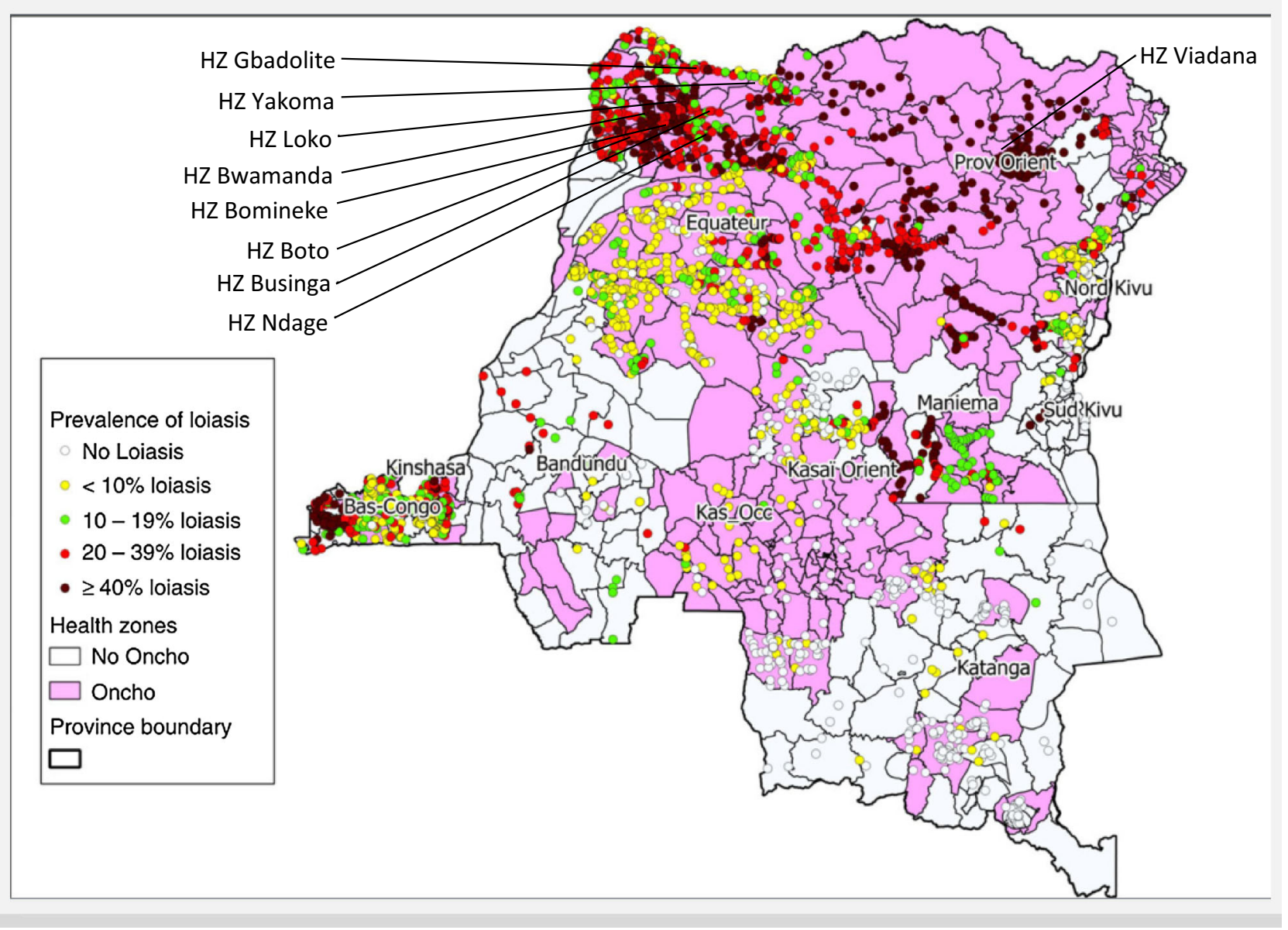

Fig. 1 Map of Democratic Republic of Congo (DRC) showing areas co-endemic for onchocerciasis and loiasis and health zones (HZs) where adverse events were reported (adapted from Makenga et al. 5)

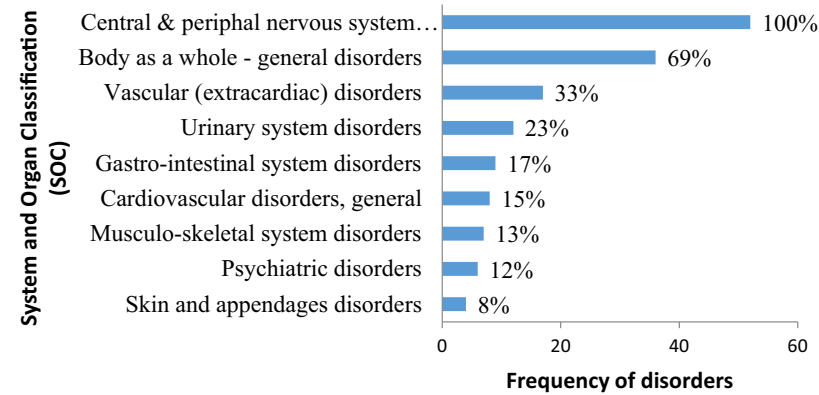

Fig. 2 Systems and organs disorders presented by patients

loiasis sites in the DRC in relation to HD areas reporting SAEs related to receiving ivermectin.

Such overlap of HD areas likely to experience SAEs supports the need for the 'point of care' diagnostic devices being developed to detect people with high levels of microfilaremia $(>30,000 \mathrm{mf} / \mathrm{ml})$ [10]. The use of such a device will allow those at higher risk of SAEs to be excluded from taking ivermectin during control exercises. However, $60 \%$ of patients had L. loa microfilariae levels
Table 2 Distribution of adverse events among central and peripheral nervous system disorders by preferred term

\begin{tabular}{lll}
\hline Preferred term & \multicolumn{2}{l}{ Frequency $(\%)$} \\
\cline { 2 - 3 } & All $(n=52)$ & PLERM $(n=23)$ \\
\hline Headache & $22(42.3)$ & $5(21.7)$ \\
Coma & $18(34.6)$ & $17(73.9)$ \\
Gait abnormal & $17(32.7)$ & $5(21.7)$ \\
Stupor & $8(15.4)$ & $7(30.4)$ \\
Dizziness & $7(13.5)$ & $0(0.0)$ \\
Vertigo & $4(7.7)$ & $1(4.3)$ \\
Fecal incontinence & $3(5.8)$ & $2(8.7)$ \\
Neurological disorder NOS & $3(5.8)$ & $0(0.0)$ \\
Convulsion & $2(3.8)$ & $1(4.3)$ \\
Aphasia & $1(1.9)$ & $0(0.0)$ \\
Ataxia & $1(1.9)$ & $0(0.0)$ \\
Facial paralysis & $1(1.9)$ & $1(4.3)$ \\
\hline AEs adverse events $N O S$ & oth
\end{tabular}

AEs adverse events, NOS not otherwise specified, PLERM probable/possible Loa loa encephalopathy temporally related to mectizan 
Table 3 Distribution of adverse events in other system organ classes, out of central and peripheral nervous system disorders, by preferred term

\begin{tabular}{lll}
\hline Preferred term & \multicolumn{2}{l}{ Frequency $(\%)$} \\
\cline { 2 - 3 } & All $(n=52)$ & PLERM $(n=23)$ \\
\hline Asthenia & $24(46.2)$ & $10(43.5)$ \\
Conjunctival hemorrhage & $17(32.7)$ & $7(30.4)$ \\
Fever & $16(30.8)$ & $6(26.1)$ \\
Back pain & $9(17.3)$ & $1(4.3)$ \\
Urinary incontinence & $9(17.3)$ & $7(30.4)$ \\
Diarrhea & $6(11.5)$ & $1(4.3)$ \\
Vomiting & $6(11.5)$ & $2(8.7)$ \\
Hypotension & $5(9.6)$ & $1(4.3)$ \\
Myalgia & $5(9.6)$ & $3(13.0)$ \\
Fatigue & $4(7.7)$ & $0(0.0)$ \\
Agitation & $3(5.8)$ & $2(8.7)$ \\
Hypertension & $3(5.8)$ & $2(8.7)$ \\
Pruritus & $3(5.8)$ & $1(4.3)$ \\
Abnormal behavior & $2(3.8)$ & $2(8.7)$ \\
Arthralgia & $2(3.8)$ & $1(4.3)$ \\
Oliguria & $2(3.8)$ & $0(0.0)$ \\
Abdominal pain & $1(1.9)$ & $0(0.0)$ \\
Angioedema & $1(1.9)$ & $1(4.3)$ \\
Personality disorder & $1(1.9)$ & $0(0.0)$ \\
Pyuria & $1(1.9)$ & $0(0.0)$ \\
Somnolence & $1(1.9)$ & $0(0.0)$ \\
\hline AEs & &
\end{tabular}

AEs adverse events, PLERM probable/possible Loa loa encephalopathy temporally related to mectizan, $S O C$ system organ class

less than the defined threshold within 1 month post-treatment, which raises the possibility of PLERM cases even with peripheral blood microfilariae less than the defined pre-treatment load in some settings. This should support the necessity of establishing a good pharmacovigilance system while implementing the use of these devices in the field to catch PLERM cases that may occur with a lower pre-treatment threshold.

It is also important to note that not all those who experienced SAEs, including coma, had microfilariae in their peripheral blood. Some had microfilariae in the cerebrospinal fluid instead, as we found in our study and Gardon et al. [17] reported from Cameroon much earlier. Therefore, the use of screening devices would have to take such variations into consideration.

Similar to reports from Cameroon by Twum-Danso and Meredith [18], SAEs began to manifest within the first few days after taking the drug, with $98 \%$ of cases showing symptoms by the fifth day (mean 2.4). Among all patients with SAEs, 5.8\% died and another 5.8\% had disabilities. Among those with PLERM, $4.4 \%$ died and $8.7 \%$ had disabilities. Early reporting and adequate care is therefore required for the sustenance of the control program. Among PLERM cases, the fatality rate found in the current study (4.4\%) was better than that shown by data from before $2003(23.5 \%)$ [16], suggesting that improvements in diagnosis and case management of $L$. loa encephalopathy between 2003 and 2013 have possibly contributed to a decrease in case fatality rate.

\section{Conclusion}

Data from the DRC pharmacovigilance system suggest ivermectin may be responsible for SAEs following MDA, with possible effects on the central and peripheral nervous system that may occur even in those with low microfilaremia concentrations in the peripheral blood. However, the distribution of these SAEs is very localized, and therefore, with adequate logistical and human resource mobilization, could be managed without disrupting the national control program. Active pharmacovigilance, including cohort event monitoring and further analytic studies, is needed to determine the proportion of SAEs and central and peripheral nervous system disorders and factors associated with the evolution towards $L$. loa encephalopathy in order to improve its management.

Acknowledgements The authors would like to thank the following healthcare workers from different health zones for reporting adverse events to the national pharmacovigilance system: Sana Pothin, Happy Ngongo Losa, John Otomba, Ruben Atandele, Jacques Dawili, Dr. Bobozo, Alain Angoy Moday, Theo Bungu Barabutu, Pascal Bagaza Ndemanza, Papa Zagba, and Juste Momboto. We are also grateful to the Mectizan ${ }^{\circledR}$ Donation Program for the availability of ivermectin and the support for passive surveillance of serious adverse events at the local level.

\section{Compliance with Ethical Standards}

Conflict of interest DN, AF, SH, DB, DM, JM, GM,CN, SM, and GT have no conflicts of interest.

Funding No external funding was received for this work.

Open Access This article is distributed under the terms of the Creative Commons Attribution-NonCommercial 4.0 International License (http://creativecommons.org/licenses/by-nc/4.0/), which permits any noncommercial use, distribution, and reproduction in any medium, provided you give appropriate credit to the original author(s) and the source, provide a link to the Creative Commons license, and indicate if changes were made. 


\section{References}

1. Hoerauf A, Pfarr K, Mand S, Debrah AY, Specht S. Filariasis in Africa-treatment challenges and prospects. Clin Microbiol Infect. 2011;17(7):977-85.

2. Molyneux DH, Bradley M, Hoerauf A, Kyelem D, Taylor MJ. Mass drug treatment for lymphatic filariasis and onchocerciasis. Trends Parasitol. 2003;19(11):516-22.

3. African Programme for Onchocerciasis Control: Meeting of National Onchocerciasis Task Forces, September 2013. Wkly Epidemiol Rec. 2013;88(50):533-44. http://www.who.int/wer/ 2013/wer8850.pdf?ua=1.

4. Kelly-Hope LA, Thomas BC, Bockarie MJ, Molyneux DH. Lymphatic filariasis in the Democratic Republic of Congo; micro-stratification overlap mapping (MOM) as a prerequisite for control and surveillance. Parasit Vectors. 2011;4:178.

5. Makenga Bof JC, Maketa V, Bakajika DK, Ntumba F, Mpunga D, Murdoch ME, Hopkins A, Noma MM, Zouré H, Tekle AH, Katabarwa MN, Lutumba P. Onchocerciasis control in the Democratic Republic of Congo (DRC): challenges in a post-war environment. Trop Med Int Health. 2015;20(1):48-62.

6. Meredith SE, Dull HB. Onchocerciasis: the First Decade of Mectizan $^{\mathrm{TM}}$ Treatment. Parasitol Today. 1998;14(12):472-4.

7. Geary TG. Ivermectin 20 years on: maturation of a wonder drug. Trends Parasitol. 2005;21(11):530-2.

8. Boussinesq M, Gardon J, Gardon-wendel N, Chippaux JP. Clinical picture, epidemiology and outcome of Loa-associated serious adverse events related to mass ivermectin treatment of onchocerciasis in Cameroon. Filaria J. 2003;2(Suppl 1):S4. doi:10.1186/1475-2883-2-S1-S4.

9. Mackenzie C, Geary T, Prichard R, Boussinesq M. Where next with Loa loa encephalopathy? Data are badly needed. Trends Parasitol. 2007;23(6):237-8.

10. D'ambrosio MV, Bakalar M, Bennuru S, Reber C, Skandarajah A, Nilsson L, Switz N, Kamgno J, Pion S, Boussinesq M, Nutman TB, Fletcher DA. Point-of-care quantification of blood-borne filarial parasites with a mobile phone microscope. Sci Transl Med. 2015;7(286):286re4.

11. Twum-danso NA. Loa loa encephalopathy temporally related to ivermectin administration reported from onchocerciasis mass treatment programs from 1989 to 2001: implications for the future. Filaria J. 2003;2(Suppl 1):S7.

12. Twum-danso NA. Serious adverse events following treatment with ivermectin for onchocerciasis control: a review of reported cases. Filaria J. 2003;2(Suppl 1):S3.

13. Boussinesq M, Gardon J, Gardon-wendel N, Kamgno J, Ngoumou P, Chippaux JP. Three probable cases of loa loa encephalopathy following ivermectin treatment for onchocerciasis. Am J Trop Med Hyg. 1998;58(4):461-9.

14. Kamgno J, Boussinesq M, Labrousse F, Nkegoum B, Thylefors BI, Mackenzie CD. Case Report: encephalopathy after Ivermectin treatment in a patient infected with Loa loa and Plasmodium spp. Am J Trop Med Hyg. 2008;78(4):546-51.

15. Kelly-hope LA, Cano J, Stanton MC, Bockarie MJ, Molyneux DH. Innovative tools for assessing risks for severe adverse events in areas of overlapping Loa loa and other filarial distributions: the application of micro-stratification mapping. Parasit Vectors. 2014;7:307.

16. Duke BO. Overview: report of a Scientific Working Group on serious adverse events following Mectizan ${ }^{\circledR}$ treatment of onchocerciasis in Loa loa endemic areas. Filaria J. 2003;2(Suppl 1):S1.

17. Gardon J, Gardon-Wendel N, Demanga-Ngangue, Kamgno J, Chippaux JP, Boussinesq M. Serious reactions after mass treatment of onchocerciasis with ivermectin in an area endemic for Loa loa infection. Lancet. 1997;350(9070):18-22. doi:10.1016/ S0140-6736(96)11094-1.

18. Twum-Danso NA, Meredith SE. Variation in incidence of serious adverse events after onchocerciasis treatment with ivermectin in areas of Cameroon co-endemic for loiasis. Trop Med Health. 2003;8(9):820-31. 